\title{
INVERSE SEMIGROUPS AS EXTENSIONS OF SEMILATTICES
}

\author{
by LIAM O'CARROLL
}

(Received 20 October, 1973)

1. Introduction. Let $S$ be an inverse semigroup with semilattice of idempotents $E$, and let $\rho$ be a congruence on $S$. Then $\rho$ is said to be idempotent-determined [2], or I.D. for short, if $(a, b) \in \rho$ and $a \in E$ imply that $b \in E$. If, further, $\rho$ is a group congruence, then clearly $\rho$ is the minimum group congruence on $S$, and in this case $S$ is said to be proper [8]. Let $T=S / \rho$.

Let $\rho$ be an I.D. congruence on $S$; the homomorphism $\rho^{\natural}$ will also be called I.D. Green [2] has given the structure of $S$ in terms of $T, E$, and certain mappings. In the case where $T$ is a group, that is when $S$ is proper, two structure theorems for $S$ have been given. One, due to the present author [5], bears some resemblance to Green's result. The other, due to McAlister [3], is extremely concrete and shows that $S$ is isomorphic to a semigroup which involves $T$ acting by order-automorphisms on a poset containing a copy of $E$ as an order-ideal.

The present paper is concerned with carrying out McAlister's programme for the case where $\rho$ is an arbitrary I.D. congruence, so that $S$ is now an arbitrary inverse semigroup; with generalising the embedding theorems for proper inverse semigroups given in $[5,6]$; and with expanding and slightly improving Green's result, using ideas from [5].

It will be found that $S$ can be embedded in a certain way in an inverse semigroup $L=L(S)$ arising from the action of $T$ on a poset by partial order-isomorphisms whose domains and ranges are order-ideals. This embedding is surjective exactly in the case where $T$ is a group, that is $S$ is proper. Furthermore, $L$ can be embedded in an inverse semigroup $\bar{L}$ arising from a similar action of $T$ on a semilattice. Thus $S$ is embedded in $\bar{L}$, a fact which overlaps with some results due to Reilly [7].

It is also shown that $S$ can be embedded in an inverse semigroup $M$, on which there is defined an I.D. congruence $\vec{\rho}$ extending $\rho$, such that each $\bar{\rho}$-class has a maximum element under the natural partial order, and $T=M / \bar{\rho}$. This is then used to yield a slight improvement in Green's theory.

Finally, the $L$-semigroups definable over an inverse semigroup $S$ are seen to form a category with initial and terminal object.

2. The embedding of $S$ in $L$. The notation and terminology of Clifford and Preston [1] will be used, and the basic results on inverse semigroups contained therein assumed. Any order-theoretic statement made about an inverse semigroup refers to the natural partial order. The identity congruence will be denoted by $i$.

The first proposition generalises a result in [8] and is implicit in [2].

PROPOSITION 2.1. Let $\rho$ be a congruence on $S$. Then $\rho$ is I.D. if and only if $\rho \cap \mathscr{R}=i$.

Proof. Suppose that $\rho$ is I.D. and let $(a, b) \in \rho \cap \mathscr{R}$. Now $\mathscr{R}$ is a left congruence, so 
that $\left(a^{-1} a, a^{-1} b\right) \in \rho \cap \mathscr{R}$. Hence $a^{-1} b \in E$, since $\rho$ is I.D. Now $a a^{-1}=b b^{-1}$, and so $b=a a^{-1} b \leqq a$; similarly, $b \leqq a$. Hence $\rho \cap \mathscr{R}=i$.

Conversely, suppose that $\rho \cap \mathscr{R}=i$ and let $(e, x) \in \rho$ where $e=e^{2}$. Then $\left(e, x x^{-1}\right) \in \rho$, so that $\left(x x^{-1}, x\right) \in \rho \cap \mathscr{R}$. Hence $x=x x^{-1} \in E$, and therefore $\rho$ is I.D.

For the remainder of the paper, $\rho$ denotes an I.D. congruence on $S$. Following Proposition 2.1, $S$ is coordinatised by the map $a \rightarrow\left(a a^{-1}, a \rho\right)$ (see [2]).

Let $\mathscr{X}$ be a poset. A non-empty subset $A$ of $\mathscr{X}$ is called an order-ideal of $\mathscr{X}$ if $b \in \mathscr{X}$ and $b \leqq a \in A$ imply that $b \in A$; and $A$ is called a subsemilattice of $\mathscr{X}$ if given $a, b \in A$ their infimum in $\mathscr{X}$, denoted $a \wedge b$, exists and is in $A$.

Let $K_{\mathscr{X}}$ be the inverse subsemigroup of $\mathscr{I}_{\mathscr{X}}$ consisting of those $\alpha \in \mathscr{I}_{\mathscr{X}}$ whose domain $\Delta \alpha$ and range $\nabla \alpha$ are order-ideals of $\mathscr{X}^{-}$, and where $\alpha$ is an order-isomorphism from $\Delta \alpha$ onto $\nabla \alpha$. We say that an inverse semigroup $T$ acts suitably on $\mathscr{X}$ if there exists a homomorphism $\phi: T \rightarrow$ $K_{x}$. In this case, for $t \in T$, we write $\Delta t$ for $\Delta(t \phi)$ and $\nabla t$ for $\nabla(t \phi)$. If $a \in \Delta t$, we let $T$ act on the left and write $t$. $a$ or $t a$ for $(t \phi) . a$. All other mappings will act on the right, as usual.

Recall that in $\mathscr{F}_{x}$, and so in $K_{x}, \alpha \leqq \beta$ if and only if (i) $\Delta \alpha \subseteq \Delta \beta$ and (ii) $\beta \mid \Delta \alpha=\alpha$.

Of necessity we follow McAlister's theory; the argument is refined or the theory generalised at those points where the fact that $T$ is no longer necessarily a group comes into play.

Proceeding as in [3], therefore, let $\left\{D_{i} \mid i \in I\right\}$ be the set of $\mathscr{D}$-classes of $S$ and pick an idempotent $f_{i} \in D_{i}$ for each $i \in I$. Denote by $H_{i}$ the $\mathscr{H}$-class containing $f_{i}$, and let $f_{i}=f_{i} \rho^{\natural}$. Further, for each $i \in I$, pick representatives $r_{i u}$ of the $\mathscr{H}$-classes contained in the $\mathscr{R}$-class of $f_{i}$ with $f_{i}$ the representative of its class; denote this set of representatives by $E_{i}$.

From now on we use $r_{i u}, r_{i v}, \ldots$ to denote elements of $E_{i}$, and $h_{i}, h_{i}^{\prime}, \ldots$ to denote elements of $H_{i}$, for some $i \in I$.

Each element of $S$ can be uniquely expressed in the form $r_{i u}^{-1} h_{i} r_{i v}$, and the idempotents of $S$ are precisely the elements $r_{i u}^{-1} r_{i u}$; they are all distinct.

Let $k_{i u}=r_{i u} \rho^{\natural}, g_{i}=h_{i} \rho^{\natural}$ and $G_{i}=H_{i} \rho^{\natural}$. By Proposition 2.1, $G_{i} \approx H_{i}$ and for fixed $i$ the elements $k_{i u}$ are all distinct.

The following trivial result, and the one derived from it by applying $\rho^{\natural}$, will be used below without comment.

Lemma 2.2. Let $a=r_{i u}^{-1} h_{i} r_{i v}$. Then $a a^{-1}=r_{i u}^{-1} r_{i u}, a^{-1} a=r_{i v}^{-1} r_{i v}$, and $f_{i} r_{i u}=r_{i w}$.

Finally, for each $i, j \in I$, let $B_{i j}=\left\{k_{j u} \mid r_{j u}^{-1} r_{j u} \leqq f_{i}\right\}$.

The next three technical lemmas which we quote are taken from [3], the second having been slightly adapted. Their proofs in [3] can be carried over without difficulty.

LEMMA 2.3. [3, Lemma 2.1.] $r_{i u}^{-1} r_{i u} \geqq r_{j v}^{-1} r_{j v}$ if and only if $G_{j} k_{j v}=G_{j} k_{j w} k_{i u}$ for some $k_{j w} \in B_{i j}$.

LEMMA 2.4. [3, Lemma 2.2.] If $k_{i u} g_{j} k_{j v} \in G_{i}$ for some $k_{i u} \in B_{j i}$ and $k_{j v} \in B_{i j}$, then $i=j$ and $k_{j v}=f_{j}$.

LEMMA 2.5. [3, Lemma 2.3.] If $k_{j v} \in B_{i j}$ and $k_{n w} \in B_{j n}$, then $G_{n} k_{n w} g_{i} k_{j v}=G_{n} k_{n u}$ for some $k_{n u} \in B_{i n}$. 
As in [3], therefore, it follows from Lemma 2.3 that the semilattice $E$ is isomorphic to the set $\mathscr{Y}=\left\{\left(i, G_{i} k_{i u}\right) \mid i \in I, k_{i u} \in E_{i} \rho^{\natural}\right\}$, where $\left(i, G_{i} k_{i u}\right) \geqq\left(j, G_{j} k_{j v}\right)$ if and only if $G_{j} k_{j v}=G_{j} k_{j w} k_{i u}$ for some $k_{j w} \in B_{i j}$.

Let $\mathscr{X}=\left\{\left(i, G_{i} x\right) \mid i \in I, x x^{-1}=f_{i}\right\}$ under the ordering $\left(i, G_{i} x\right) \geqq\left(j, G_{j} y\right)$ if and only if $G_{j} y=G_{j} z x$ for some $z \in B_{i j} . \quad T$ acts on $\mathscr{X}$ by partial transformations as follows:

$$
\Delta t=\left\{\left(i, G_{i} x\right) \in \mathscr{X} \mid x^{-1} x \leqq t^{-1} t\right\},
$$

and, for $\left(i, G_{i} x\right) \in \Delta t, t .\left(i, G_{i} x\right)=\left(i, G_{i} x t^{-1}\right)$.

LemMA 2.6. $\mathscr{X}$ is a poset, and $\mathscr{Y}$ is a subsemilattice and order-ideal of $\mathscr{X} . T$ acts suitably on $\mathscr{X}$ and $\mathscr{X}=T \mathscr{Y}$. For each $t \in T, \mathscr{Y} \cap t(\mathscr{Y} \cap \Delta t) \neq \square$.

Proof. Note that Lemmas 2.3, 2.4 and 2.5 hold. The argument given in [3] prior to Lemma 2.4 there, adapted slightly by noting that $\bar{f}_{j} \in B_{j j}$ and $g_{j}=g_{j} \bar{f}_{j}$, shows that $\geqq$ is a well-defined relation on $\mathscr{X}$. The relevant parts of the proof of [3, Lemma 2.4], with a similar small adaptation, show that $\geqq$ is reflexive, transitive and antisymmetric, and that $\mathscr{Y}$ is a subsemilattice and order-ideal of $\mathscr{X}$.

Let $\left(i, G_{i} x\right) \in \mathscr{X}$, where $x^{-1} x \leqq t^{-1} t$; then $\left(x t^{-1}\right)\left(x t^{-1}\right)^{-1}=x t^{-1} t x^{-1}=x x^{-1}=f_{i}$. Hence $t .\left(i, G_{i} x\right) \in \mathscr{X}$. Suppose further that $\left(j, G_{j} y\right) \in \mathscr{X}$, where $\left(j, G_{j} y\right) \leqq\left(i, G_{i} x\right)$. Then $y=g_{j} z x$ for some $z \in B_{i j}$, so that

$$
y^{-1} y=x^{-1} z^{-1} g_{j}^{-1} g_{j} z x \leqq x^{-1} x \leqq t^{-1} t .
$$

Hence $\left(j, G_{j} y\right) \in \Delta t$, and therefore $\Delta t$ is an order-ideal of $\mathscr{X}$. Moreover $t .\left(i, G_{i} x\right) \geqq t .\left(j, G_{j} y\right)$, and $t .\left(i, G_{i} x\right) \in \Delta t^{-1}$ since $\left(x t^{-1}\right)^{-1} x t^{-1} \leqq\left(t^{-1}\right)^{-1} t^{-1}$.

Hence $\nabla t \subseteq \Delta t^{-1}$. On the other hand if $\left(i, G_{i} z\right) \in \Delta t^{-1}$, then $z z^{-1}=f_{i}$ and $z^{-1} z \leqq t t^{-1}$. Let $w=z t$; then $w w^{-1}=z t t^{-1} z^{-1}=z z^{-1}=f_{i}, w^{-1} w \leqq t^{-1} t$, and $z=w t^{-1}$. Hence $\left(i, G_{i} w\right) \in \Delta t$ and $t .\left(i, G_{i} w\right)=\left(i, G_{i} z\right), t^{-1}\left(i, G_{i} z\right)=\left(i, G_{i} w\right)$.

Thus $\nabla t=\Delta t^{-1}$ is an order-ideal, and $t$ is a partial order-isomorphism with domain $\Delta t$ and range $\nabla t$, having inverse $t^{-1}$. If $s \in T$, it is easily shown that $\Delta(t s)=s^{-1}(\nabla s \cap \Delta t)=$ $\Delta(t \circ s)$, and clearly therefore $T$ acts suitably on $\mathscr{X}$. $T \mathscr{Y}$.

Let $\left(i, G_{i} x\right) \in \mathscr{X}$. Then $\left(i, G_{i} f_{i}\right) \in \mathscr{Y} \cap \Delta x^{-1}$, and $x^{-1} \cdot\left(i, G_{i} \bar{f}_{i}\right)=\left(i, G_{i} x\right)$. Thus $\mathscr{X}=$

Let $t \in T$, where $t=k_{i u}^{-1} g_{i} k_{i v}$, say. Then $\left(i, G_{i} k_{i u}\right) \in \mathscr{Y} \cap t(\mathscr{Y} \cap \Delta t)$, since $t .\left(i, G_{i} k_{i v}\right)=$ $\left(i, G_{i} k_{i u}\right)$.

Suppose now that we are given a poset $\mathscr{X}$ containing a subsemilattice and order-ideal $\mathscr{Y}$, and an inverse semigroup $T$, which together have the properties listed in the statement of Lemma 2.6.

LEMMA 2.7. For each $t \in T, \mathscr{Y} \cap t\left(\mathscr{Y} \cap t^{-1}\left(\mathscr{Y} \cap \Delta t^{-1}\right)\right) \neq \square$.

Proof. By hypothesis there exists $a \in \mathscr{Y} \cap \Delta t^{-1}$ such that $b=t^{-1} a \in \mathscr{Y}$. Then $b \in$ $\nabla t^{-1}=\Delta t$, and $t b=t t^{-1} a=a$. Hence $a \in \mathscr{Y} \cap t\left(\mathscr{Y} \cap t^{-1}\left(\mathscr{Y} \cap \Delta t^{-1}\right)\right)$. 
Define $L=L(T, \mathscr{X}, \mathscr{Y})$ to be

$$
\left\{(a, t) \mid t \in T, a \in \mathscr{Y} \cap t\left(\mathscr{Y} \cap t^{-1}\left(\mathscr{Y} \cap \Delta t^{-1}\right)\right)\right\}
$$

under the multiplication

$$
(a, t)(b, s)=\left(t\left(t^{-1} a \wedge b\right), t s\right) .
$$

By Lemma 2.7, for each $t \in T$ there exists $(a, t) \in L$.

Whenever $T$ is a group, that is whenever $S$ is proper and $\rho$ is the minimum group congruence on $S$, then $T$ acts on $\mathscr{X}$ by order-automorphisms, and $L=P(T, \mathscr{X}, \mathscr{Y})$ as defined in [3].

Lemma 2.8. Let $a \in \mathscr{X}, t \in T$. Then $(a, t) \in L$ if and only if (i) $a \in \mathscr{Y} \cap \Delta t^{-1}$ and (ii) $t^{-1} a \in \mathscr{Y}$.

Proof. This follows easily from the elementary observation that $a \in \nabla t$ if and only if $a \in \Delta t^{-1}$, and then $a=t t^{-1} a$.

CoRollary 2.9. Suppose $(a, t) \in L$ and $s \geqq t$. Then $(a, s) \in L$.

Proof. Since $s^{-1} \geqq t^{-1}, \Delta t^{-1} \subseteq \Delta s^{-1}$ and $s^{-1} a=t^{-1} a$. The result now follows from Lemma 2.8 .

THEOREM 2.10. L is an inverse semigroup. If $\pi_{2}: L \rightarrow T$ is the second projection $(a, t) \mapsto t$, then $\pi_{2}$ is an I.D. surjective homomorphism.

Proof. Let $(a, t),(b, s) \in L$. By Lemma 2.8, $t^{-1} a \in \mathscr{Y}$ so that $t^{-1} a \wedge b$ exists and is in $\mathscr{Y}$. Since $\nabla t^{-1}$ and $\Delta s^{-1}$ are order-ideals and $t^{-1} a \in \nabla t^{-1}, t^{-1} a \wedge b \in \nabla t^{-1} \cap \Delta s^{-1}$. Hence $t\left(t^{-1} a \wedge b\right) \in \Delta s^{-1} t^{-1}=\Delta(t s)^{-1}$. Moreover $t\left(t^{-1} a \wedge b\right) \leqq t t^{-1} a=a \in \mathcal{Y}$, so that $t\left(t^{-1} a \wedge b\right) \in \mathscr{Y}$. Further, $s^{-1} t^{-1} t\left(t^{-1} a \wedge b\right)=s^{-1}\left(t^{-1} a \wedge b\right) \leqq s^{-1} b \in \mathscr{Y}$, by Lemma 2.8 . By Lemma 2.8 again, therefore,

$$
(a, t)(b, s)=.\left(t\left(t^{-1} a \wedge b\right), t s\right) \in L,
$$

and $L$ is closed under multiplication.

Let $(c, r) \in L$. It is easily seen that

$$
(a, t)[(b, s)(c, r)]=[(a, t)(b, s)](c, r)
$$

if and only if

$$
t\left(t^{-1} a \wedge s\left(s^{-1} b \wedge c\right)\right)=t s\left(s^{-1}\left(t^{-1} a \wedge b\right) \wedge c\right)
$$

that is, if and only if

$$
t^{-1} a \wedge s\left(s^{-1} b \wedge c\right)=s\left(s^{-1}\left(t^{-1} a \wedge b\right) \wedge c\right)
$$


that is, if and only if

$$
s^{-1}\left(t^{-1} a \wedge s\left(s^{-1} b \wedge c\right)\right)=s^{-1}\left(t^{-1} a \wedge b\right) \wedge c .
$$

Now the left hand side of $(2) \leqq s^{-1}\left(t^{-1} a \wedge s s^{-1} b\right)=s^{-1}\left(t^{-1} a \wedge b\right)$; and further, the left hand side of $(2) \leqq s^{-1} s\left(s^{-1} b \wedge c\right)=s^{-1} b \wedge c \leqq c$. Hence the left hand side of $(2) \leqq$ the right hand side of (2). Applying $s$ on the left, we deduce that the left hand side of $(1) \leqq$ the right hand side of $(1)$.

On the other hand, the right hand side of $(1) \leqq s\left(s^{-1} b \wedge c\right)$; and further, the right hand side of $(1) \leqq s s^{-1}\left(t^{-1} a \wedge b\right)=t^{-1} a \wedge b \leqq t^{-1} a$. Hence the right hand side of $(1) \leqq$ the left hand side of (1). Equality follows, so that the multiplication is associative.

It is easily seen that the set of idempotents $\mathscr{E}$ of $L$ is given by $\mathscr{E}=\left\{(a, t) \mid t=t^{2}\right\}$ and that the elements of $\mathscr{E}$ commute. In fact, if $(a, t)$ and $(b, s) \in \mathscr{E}$, then

$$
(a, t)(b, s)=(a \wedge b, t s)
$$

Some routine checking then shows that $L$ is an inverse semigroup with $(a, t) \in L$ having inverse $\left(t^{-1} a, t^{-1}\right)$. Note that $(a, t)(a, t)^{-1}=\left(a, t t^{-1}\right)$, so that if $(a, t) R(b, s)$ then $a=b$. Clearly $\pi_{2}$ is a surjective homomorphism, and if further $(a, t) \pi_{2}=(b, s) \pi_{2}$, then $t=s$. Hence $\pi_{2}$ is I.D., by Proposition 2.1.

REMARK. Let $(a, t),(b, s) \in \mathscr{E}$; then their product $(a \wedge b, t s) \in \mathscr{E}$. Since $t s \leqq t$, it follows from Corollary 2.9 and Theorem 2.10 that $(a \wedge b, t) \in \mathscr{E}$.

Define the projection $\pi_{1}: \mathscr{E} \rightarrow \mathscr{Y}$ by $(a, t) \pi_{1}=a$. Then $\pi_{1}$ is a homomorphism with range $\{a \in \mathscr{Y} \mid a \in \Delta r$ for some $r \in T\}$. If $t \neq s$, then we may assume that $t s<t$, and $(a \wedge b, t) \pi_{1}=(a \wedge b, t s) \pi_{1}$.

Hence $\pi_{1}$ is injective if and only if $T$ has exactly one idempotent, that is if and only if $T$ is a group. In this case, $T$ acts by order-automorphisms on $\mathscr{X}$ and $\pi_{1}$ is surjective.

We now have the main theorem of this section, which describes how $S$ is embedded in the corresponding $L$.

As before, let $S$ be an inverse semigroup with semilattice of idempotents $E$, and let $\rho$ be an I.D. congruence on $S$. Suppose that $\mathscr{X}, \mathscr{Y}$ and $T$ are as defined prior to Lemma 2.6. Let $L=L(S)$, where $L(S)=L(T, \mathscr{X}, \mathscr{Y})$, and define the map $\psi: S \rightarrow L$ as follows:

$$
\left(r_{i u}^{-1} h_{i} r_{i v}\right) \psi=\left(\left(i, G_{i} k_{i u}\right), k_{i u}^{-1} g_{i} k_{i v}\right)
$$

THEOREM 2.11. $\psi$ is an injective homomorphism such that $\psi \pi_{2}=\rho^{\sharp}$. For each $a \in \mathscr{Y}$ there exists $(a, t) \in L$ with the following property:

$$
(a, s) \in L \text { and } s \leqq t \text { imply that } s=t:
$$

and $S \psi$ is the set of all such $(a, t)$. Moreover, given $a \in \mathscr{Y}$, then $(a, s) \in L$ if and only if there exists $(a, t) \in S \psi$ with $t \leqq s$. 
Proof. The last paragraph of the proof of Lemma 2.6 shows that $\psi$ indeed maps into $L$. The argument given prior to [3, Lemma 2.5] shows that $\psi$ is injective, and clearly $\psi \pi_{2}=\rho^{q}$.

Let

$$
p=r_{i u}^{-1} h_{i} r_{i v}, q=r_{j x}^{-1} h_{j} r_{j y}
$$

be elements of $S$. Following the first part of the proof of [3, Lemma 2.5],

$$
r_{i v}^{-1} r_{i v} r_{j x}^{-1} r_{j x}=r_{n w}^{-1} r_{n w}, \text { for some } r_{n w} \in E_{n},
$$

where

$$
r_{n w} r_{i v}^{-1} h_{i}^{-1} r_{i u}=h_{n} r_{n z} \text { for some } r_{n z} \in E_{n}, h_{n} \in H_{n} \text {. }
$$

Further,

$$
p q=r_{n z}^{-1} h_{n}^{\prime} r_{n c} \text { for some } r_{n c} \in E_{n}, h_{n}^{\prime} \in H_{n} .
$$

Thus $\left(n, G_{n} k_{n z}\right)$ is the first coordinate of $(p q) \psi$, and

$$
\left(n, G_{n} k_{n z}\right)=\left(n, G_{n} k_{n w} k_{i v}^{-1} g_{i}^{-1} k_{i u}\right) .
$$

Now

$$
k_{n w}^{-1} k_{n w} \leqq k_{i v}^{-1} k_{i v}=\left(k_{i u}^{-1} g_{i} k_{i v}\right)^{-1}\left(k_{i u}^{-1} g_{i} k_{i v}\right)
$$

so that

$$
\left(n, G_{n} k_{n z}\right)=k_{i u}^{-1} g_{i} k_{i v} \cdot\left(n, G_{n} k_{n w}\right)=p \rho^{\natural} \cdot\left\{\left(i, G_{i} k_{i v}\right) \wedge\left(j, G_{j} k_{j x}\right)\right\} .
$$

As seen in the last paragraph of the proof of Lemma 2.6, $\left(i, G_{i} k_{i v}\right)=\left(p \rho^{\natural}\right)^{-1}\left(i, G_{i} k_{i u}\right)$, and it follows that $(p q) \psi$ and $p \psi . q \psi$ have the same first coordinate. Their second coordinates are also equal, so that $\psi$ is a homomorphism.

Given $a=\left(i, G_{i} k_{i u}\right) \in \mathscr{Y}$ therefore, take $g_{i} \in G_{i}$ and $k_{i v} \in E_{i} \rho^{\natural}$. Letting $t=k_{i u}^{-1} g_{i} k_{i v}$, it follows that $(a, t) \in S \psi$. On the other hand, by Lemma $2.8,\left(\left(i, G_{i} k_{i u}\right), t\right) \in L$ if and only if $k_{i u}^{-1} k_{i u} \leqq t t^{-1}$ and $t^{-1}\left(i, G_{i} k_{i u}\right)=\left(i, G_{i} k_{i w}\right)$ for some $k_{i w} \in E_{i} \rho^{q}$. The latter conditions hold if and only if $k_{i u}^{-1} k_{i u} \leqq t t^{-1}$ and $k_{i u} t=g_{i}^{\prime} k_{i w}$, for some $g_{i}^{\prime} \in G_{i}$. that

Let $m=k_{i u}^{-1} g_{i}^{\prime} k_{i w}$; then $\left(\left(i, G_{i} k_{i u}\right), m\right) \in S \psi$. If $m \leqq t$, then $m=m m^{-1} t=k_{i u}^{-1} k_{i u} t$, so

$$
g_{i}^{\prime} k_{i w}=k_{i u} m=k_{i u} t ; \quad \text { also } \quad k_{i u}^{-1} k_{i u}=m m^{-1} \leqq t t^{-1} .
$$

Conversely, if $k_{i u} t=g_{i}^{\prime} k_{i w}$ then $m m^{-1} t=k_{i u}^{-1} k_{i u} t=k_{i u}^{-1} g_{i}^{\prime} k_{i w}=m$, so that $m \leqq t$.

Suppose now that $\left(\left(i, G_{i} k_{i u}\right), m^{\prime}\right) \in S \psi$, where $m^{\prime} \leqq m$. Then $m^{\prime}=m^{\prime} m^{\prime-1} m=$ $k_{i u}^{-1} k_{i u} m=m$. Since to any $(a, t) \in L$ there corresponds $(a, s) \in S \psi$ with $s \leqq t$, this suffices to complete the proof. 
COROLLARY 2.12. $\psi$ is surjective if and only if $S$ is proper and $\rho$ is the minimum group congruence on $S$.

Proof. Suppose that $\psi$ is surjective, and let $t, s \in T$ with $t \leqq s$. As noted prior to Lemma 2.8, $(a, t) \in L$ for some $a \in \mathscr{Y}$. By Corollary $2.9,(a, s) \in L$. Since $L=S \psi$ by hypothesis, it follows from Theorem 2.11 that $t=s$. Hence each element of $T$ is maximal in $T$, so that $T$ is a group. As noted in $\S 1$, this implies that $S$ is proper and that $\rho$ is necessarily the minimum group congruence on $S$.

The converse has been proved in [3].

Not every semigroup $L(T, \mathscr{X}, \mathscr{Y})$ is of the form $L(S)$ for some inverse semigroup $S$. However one can give necessary and sufficient conditions that this should be so.

Finally we note that $[3$, Theorem 2.7] has an obvious generalisation which we will not state here.

3. Two other embedding theorems. We now generalise two embedding theorems for proper inverse semigroups (see $[5,6])$.

Let $\mathscr{X}, \mathscr{Y}$ and $T$ have the properties listed in the statement of Lemma 2.6 , and let $L=$ $L(T, \mathscr{X}, \mathscr{Y})$.

Define $\bar{X}$ to be the set of (non-empty) order-ideals $A$ of $\mathscr{X}$ such that $A \subseteq t(\mathscr{Y} \cap \Delta t)$ for some $t \in T$. For each $a \in \mathscr{X}$, let $\bar{a}=\{b \in \mathscr{X} \mid b \leqq a\}$.

Lemma 3.1. $(\bar{X}, \cap)$ is a semilattice on which $T$ acts suitably. The map $j: a \mapsto \bar{a}$ is an order-isomorphic embedding of $\mathscr{X}$ in $\bar{X}$ which preserves the action of $T$, and $\bar{X}$ is a conditional V-completion for $\mathscr{X} j$.

Proof. Let $s, t \in T$. It is clear that $\mathscr{Y} \cap \Delta t$ is a non-empty order-ideal and subsemilattice of $\mathscr{X}$.

Since $\nabla t$ is an order-ideal of $\mathscr{X}$ and since $t$ is an order-isomorphism on $\Delta t$, it follows that $t(\mathscr{Y} \cap \Delta t)$ is a subsemilattice and order-ideal of $\mathscr{X}$. Moreover, $\mathscr{Y} \cap t(\mathscr{Y} \cap \Delta t) \neq$

Let $a \in A \in \bar{X}$ where $A \subseteq t(\mathscr{Y} \cap \Delta t)$, and $b \in B \in \bar{X}$, where $B \subseteq s(\mathscr{Y} \cap \Delta s)$. Let $c \in$ $\mathscr{Y} \cap t(\mathscr{Y} \cap \Delta t), d \in \mathscr{Y} \cap s(\mathscr{Y} \cap \Delta s)$. Then $a \wedge c$ exists in $\mathscr{X}$ and lies in $\mathscr{Y} \cap t(\mathscr{Y} \cap \Delta t)$; similarly $b \wedge d$ exists in $\mathscr{X}$ and lies in $\mathscr{Y} \cap s(\mathscr{Y} \cap \Delta s)$. Hence $e=(a \wedge c) \wedge(b \wedge d)$ exists in $\mathscr{X}$ and lies in $\mathscr{Y}$. Since $e$ is a common lower bound of $a$ and $b, e \in A \cap B$. Thus $A \cap B \neq \square$, and it easily follows that $A \cap B \in \bar{X}$.

For each $t \in T$, let $\bar{\Delta} t=\{A \in \overline{\mathscr{X}} \mid A \subseteq \Delta t\}$; for example, $t^{-1}\left(\mathscr{Y} \cap \Delta t^{-1}\right) \in \bar{\Delta} t$. Clearly $\bar{\Delta} t$ is an order-ideal of $\bar{X}$. For each $A \in \bar{\Delta} t$, define $t A$ to be the set $\{t a \mid a \in A\}$. Then $t A$ is an order-ideal of $\mathscr{X}$ and if $r \in T$ is such that $A \subseteq r(\mathscr{Y} \cap \Delta r)$, then $t A \subseteq \operatorname{tr}(\mathscr{Y} \cap \Delta(t r))$. Hence $t A \in \bar{X}$, and $t A \subseteq \bar{\Delta} t^{-1}$. On the other hand, if $B \in \bar{\Delta} t^{-1}$, then $t^{-1} B \in \bar{\Delta} t$ and $t t^{-1} B=B$. Clearly, therefore, $t$ is an order-isomorphism with domain $\overline{\Delta t}$ and range $\bar{\nabla} t=\bar{\Delta} t^{-1}$. Given $A \in \bar{X}$ and $s \in T, A \in \bar{\Delta}(t s)$ if and only if $A \subseteq \Delta(t s)$; that is, if and only if $A \subseteq \Delta s$ and $s A \subseteq$ $\Delta s^{-1} \cap \Delta t$. It easily follows that $T$ acts suitably on $\overline{\mathscr{X}}$. 
Let $a \in \mathscr{X}=T \mathscr{Y}$. Then $a=s b$ for some $s \in T$ and $b \in \mathscr{Y} \cap \Delta s$. Hence $\bar{a}$ is an orderideal of $\mathscr{X}$ such that $\bar{a} \subseteq s(\mathscr{Y} \cap \Delta s)$. Let $t \in T$. Then $a \in \Delta t$ if and only if $\bar{a} \in \bar{\Delta} t$, and in this case $\overline{t a}=t \cdot \bar{a}$.

The rest of the result follows from the proof of [6, Lemma 1.2].

Following Lemma 3.1, let $A \in \bar{X}$, where $A \subseteq t(\mathscr{Y} \cap \Delta t)$ say. Then $A \in \bar{\nabla} t$, so that $A=t B$ for some $B \in \bar{\Delta} t$. Hence $\bar{X}=T \bar{X}$, and it follows from Lemmas 2.6 and 3.1 that we can form $\bar{L}=L(T, \overline{\mathscr{X}}, \overline{\mathscr{X}})$.

THEOREM 3.2. The map $k:(a, t) \mapsto(\bar{a}, t)$ is an injective homomorphism from $L$ into $L$.

Proof. Let $(a, t) \in L$. By Lemma 2.8, $a \in \mathscr{Y} \cap \Delta t^{-1}$ and $t^{-1} a \in \mathscr{Y}$. Hence $\bar{a} \in \overline{\mathscr{X}} \cap \Delta t^{-1}$ and $t^{-1} \cdot \bar{a} \in \overline{\mathscr{X}}$. Thus $k$ maps into $\bar{L}$ and clearly it is injective.

Let $(b, s) \in L$. Then

$$
\left.\overline{t\left(t^{-1} a \wedge b\right)}=t \cdot \overline{\left(t^{-1} a \wedge b\right)}=t \overline{\left(t^{-1} a\right.} \cap \bar{b}\right)=t\left(t^{-1} \cdot \bar{a} \cap \bar{b}\right),
$$

and it follows that $k$ is a homomorphism.

By Theorems 2.11 and 3.2, any inverse semigroup $S$ can be embedded in an $L(T, \mathscr{X}, \mathscr{Y})$ and so in $L(T, \bar{X}, \bar{X})$. For results of a similar nature, see [7, Propositions 1.4 and 3.6].

Now let $S$ be an inverse semigroup with semilattice of idempotents $E$, let $\rho$ be an I.D. congruence on $S$, and let $T=S / \rho$. Then $\alpha=\rho^{\natural}$ is an isotone homomorphism onto $T$. In

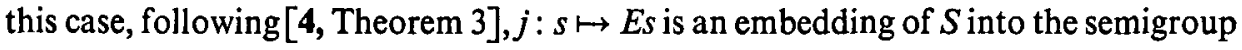

$$
M=\left\{E X \mid \text { there exists } s^{\prime} \in S \text { such that } \square \neq X \subseteq s^{\prime} \rho\right\},
$$

where the operation on $M$ is set multiplication, and $\beta: E X \mapsto s^{\prime} \rho$ is a homomorphism from $M$ onto $T$ with $\alpha=j \beta$. Moreover, $M$ is a partially ordered semigroup under inclusion and if $\bar{\rho}=\beta \circ \beta^{-1}$, then each $\bar{\rho}$-class has a maximum element.

Recall that $E W=W E$ for any non-empty subset $W$ of $S$.

THEOREM 3.3. $M$ is an inverse semigroup, and inclusion is the natural partial order on $M$. Moreover, $\bar{\rho}$ is I.D..

Proof. If $Y \subseteq S$, let $Y^{-1}=\left\{y^{-1} \mid y \in Y\right\}$. In[9], Schein showed that

$$
C=\left\{E X \mid \square \neq X \subseteq S ; X X^{-1}, X^{-1} X \subseteq E\right\}
$$

is an inverse semigroup under set multiplication, with $Y \in C$ having inverse $Y^{-1}$ (see the Note following Theorem 1 in [5]).

If $Y \in M$ then $Y^{-1} \in M$, and it follows that $M$ is an inverse subsemigroup of $C$.

Suppose $F=E X \in M$, where $\square \neq X \subseteq s \rho$ for some $s \in S$. If $F=F^{2}$, then $E X=E X^{2}$. Applying $\rho^{\natural}$, we deduce that $E \rho^{\natural} \cdot s \rho^{\natural}=E \rho^{\natural} . s^{2} \rho^{\natural}$. Hence $s \rho^{\natural}=s^{2} \rho^{\natural}$, so that $s \rho \subseteq E$ since $\rho$ is I.D.. Therefore $F \subseteq E$.

Let $Y, Z \in M$, where $Y=Y Y^{-1} Z$. By the preceding paragraph $Y Y^{-1} \subseteq E$, and $E Z=$ $Z$. Hence $Y \subseteq Z$. 
Conversely, if $Y \subseteq Z$, then $Y=Y Y^{-1} Y \subseteq Y Y^{-1} Z$, while $Y^{-1} Z \subseteq Z^{-1} Z \subseteq E$; hence $Y Y^{-1} Z \subseteq Y$. It follows that inclusion is the natural partial order.

If $s \in S$, then $E . s \rho$ is the maximum element in its $\bar{\rho}$-class, and $(E . s \rho) \bar{\rho}=\{E W \mid \square \neq W \subseteq s \rho\}$. Suppose some $E W$ in $(E . s \rho) \vec{\rho}$ is idempotent. Then, as shown above, $W \subseteq E$ and so $s \rho \subseteq E$, since $\rho$ is I.D. Hence $\bar{\rho}$ is I.D.

Green [2] has shown that there exists a maximum I.D. congruence on an inverse semigroup. Clearly $\rho$ is the maximum I.D. congruence on $S$ if and only if $\bar{\rho}$ is the maximum I.D. congruence on $M$. As in [5], $S j$ is the set of V-irreducible elements of $M$.

Let $a \in S$. In $M, a j=E a \subseteq E . a \rho$. Since inclusion is the natural partial order on $M$, by Theorem 3.3, Ea $=E a \cdot a^{-1} E \cdot a \rho=E a a^{-1} \cdot a \rho$.

The coordinatisation $a \mapsto\left(a a^{-1}, a \rho\right)$ of $S$ can be replaced by $a \mapsto\left(E a a^{-1}, a \rho\right)$, and as seen above the latter has an interpretation in terms of set multiplication.

Let $M(E)$ be the set of non-empty order-ideals of $E$ under set multiplication. As seen in [5], $M(E)$ is a semilattice in which $E$ is embedded by the map $e \mapsto E e$.

Let $H=E . a \rho$. It follows from Theorem 3.3 that $\phi_{a \rho}: F \mapsto H F H^{-1}$ is an endomorphism of $M(E)$. If $b \in S$, then $E a b(a b)^{-1}=E a a^{-1} \wedge\left(E b b^{-1}\right) \phi_{a \rho}$.

Hence in Green's theory [2, third section] we can replace the endomorphism $\phi(e, t)$ of $E$ by the endomorphism $\phi_{t}$ of $M(E)$, where the latter depends on only one parameter. However, it is extremely doubtful if a corresponding reaxiomatisation would present any real gain.

The above considerations generalise part of the theory of [5].

4. The category of $L$-semigroups over an inverse semigroup. In this final section we show that the $L$-semigroups definable over an inverse semigroup $S$ form a category with initial and terminal object. Since the details are entirely straightforward, they are omitted.

Suppose $\rho_{1}$ and $\rho_{2}$ are I.D. congruences on $S$ such that $\rho_{1} \subseteq \rho_{2}$. For $i=1,2$, given the I.D. congruence $\rho_{i}$ let $\mathscr{X}_{i}, \mathscr{Y}_{i}$ and $T_{i}$ be as defined prior to Lemma 2.6; put $L_{i}=L\left(T_{i}, \mathscr{X}_{i}, \mathscr{Y}_{i}\right)$ and let $\psi_{i}: S \rightarrow L_{i}$ be the corresponding embedding.

There is induced a unique homomorphism $\eta: T_{1} \rightarrow T_{2}$ such that $\rho_{1}^{\natural} \eta=\rho_{2}^{\natural}$. In turn $\eta$ defines a map $\mu: \mathscr{X}_{1} \rightarrow \mathscr{X}_{2}$ as follows: for $\left(i, G_{i} x\right) \in \mathscr{X}_{1},\left(i, G_{i} x\right) \mu=\left(i,\left(G_{i} x\right) \eta\right)$. Then $\mu$ has the following properties:

(i) $\mu$ is isotone, $\mathscr{Y}_{1} \mu \subseteq \mathscr{Y}_{2}$ and $\mu \mid \mathscr{Y}_{1}$ is a semilattice homomorphism; and

(ii) for each $t \in T_{1}$ and $a \in \Delta t,(\Delta t) \mu \subseteq \Delta(t \eta)$ and $(t a) \mu=t \eta$. $a \mu$.

The maps $\eta$ and $\mu$ define a map $\alpha: L_{1} \rightarrow L_{2}$ by

$$
, t) \alpha=(a \mu, t \eta)
$$

and $\alpha$ is a homomorphism such that $\psi_{1} \alpha=\psi_{2}$.

The semigroups $L_{1}$ together with the homomorphisms $\alpha$ form the objects and morphisms, respectively, of a category, which we call the category of $L$-semigroups over $S$. It has an initial object $L_{0}$ corresponding to the minimum I.D. congruence $i$, and a terminal object $L_{\infty}$ corresponding to the maximum I.D. congruence $\tau$ (see [2]). 
We note that in $L_{0}, T=S$ and we can take $\mathscr{X}=\mathscr{Y}=E$, where $E$ is the semilattice of idempotents of $S$. For $t \in S, \Delta t=\left\{e \in E \mid e \leqq t^{-1} t\right\}$ and for $e \in \Delta t, t . e=t e t^{-1}$. Then $L_{0}=\left\{(e, t) \mid e \leqq t t^{-1}\right\}$, where in $L_{0},(e, t)(f, s)=\left(\right.$ etf $\left.t^{-1}, t s\right)$.

On the other hand, suppose, for $i=1,2$, we are given a poset $\mathscr{X}_{i}$ having a subsemilattice and order-ideal $\mathscr{Y}_{i}$ and an inverse semigroup $T_{i}$ having the properties listed in the statement of Lemma 2.6 and let $L_{i}=L\left(T_{i}, \mathscr{X}_{i}, \mathscr{Y}_{i}\right)$. Let $\eta: T_{1} \rightarrow T_{2}$ be a homomorphism and $\mu: \mathscr{X}_{1} \rightarrow$ $\mathscr{X}_{2}$ a map satisfying the properties (i) and (ii) above. Then the map $\alpha: L_{1} \rightarrow L_{2}$ defined by $(a, t) \alpha=(a \mu, t \eta)$ is a homomorphism. For an analogous characterisation of homomorphisms between $P$-semigroups, see [3].

\section{REFERENCES}

1. A. H. Clifford and G. B. Preston, The algebraic theory of semigroups, Vols. I and II, Math. Surveys of the American Math. Soc. 7 (Providence, R.I., 1961 and 1967).

2. D. G. Green, Extensions of a semilattice by an inverse semigroup, Bull. Austral. Math. Soc. 9 (1973), 21-31.

3. D. B. McAlister, Groups, semilattices and inverse semigroups II, Trans. Amer. Math. Soc.; to appear.

4. L. O'Carroll, A class of congruences on a posemigroup, Semigroup Forum 3 (1971), 173-179.

5. L. O'Carroll, Reduced inverse and partially ordered semigroups, J. London Math. Soc. (2), 9 (1974), 293-301.

6. L. O'Carroll, Embedding theorems for proper inverse semigroups, J. of Algebra; submitted.

7. N. R. Reilly, Inverse semigroups of partial transformations and $\theta$-classes, Pac. J. Math. 41 (1972), 215-235.

8. T. Saito, Proper ordered inverse semigroups, Pac. J. Math. 15 (1965), 649-666.

9. B. M. Schein, Completions, translational hulls, and ideal extensions of inverse semigroups, Czech. Math. J. 23 (98) (1973), 575-610.

The Mathematical Institute

EDINBURGH EHI $1 \mathrm{HZ}$ 